\title{
Stress and associated factors with received and needed support in medical students during COVID-19 pandemic: a multicenter study
}

Tidarat Puranachaikere ${ }^{1}$, Somboon Hataiyusuk ${ }^{1}$, Rungarun Anupansupsai , Supinya In-iww', Nattha Saisavoey ${ }^{1}$, Tanawat Techapanuwat ${ }^{1}$, Fasinee Arunrodpanya ${ }^{2}$, Chanchai Charonpongsuntorn ${ }^{3}$, Pakawat Wiwattanaworaset ${ }^{4}$, Areerat Siripongpan ${ }^{5}$, Wisarat Pruttithavorn ${ }^{6}$, Chayut Wonglertwisawakorn ${ }^{6}$, Pichaya Pojanapotha ${ }^{7}$, Butsayanart Rueangrong ${ }^{8}$, Nalinee Pattrakornkul ${ }^{9}$, Nontaphon Piyawattanametha ${ }^{10}$, Siriwan Piyawattanametha $^{10}$ and Dheeravut Ratanapichayachai ${ }^{11}$

${ }^{1}$ Faculty of Medicine, Siriraj Hospital, Mahidol University, Bangkok, ${ }^{2}$ Faculty of Medicine, Naresuan University, Phitsanulok, ${ }^{3}$ Faculty of Medicine, Srinakharinwirot University, Nakhon Nayok, ${ }^{4}$ Faculty of Medicine, Prince of Songkla University, Songkhla, Institute of Medicine, Suranaree University of Technology, Nakhon Ratchasima, ${ }^{6}$ Faculty of Medicine, Vajira Hospital, Navamindradhiraj University, Bangkok, ${ }^{7}$ Faculty of Medicine, Chiangmai University, Chiang Mai, ${ }^{8}$ Ratchaburi Hospital, Ratchaburi, ${ }^{9}$ Faculty of Medicine, Burapha University, Chon Buri, ${ }^{10}$ Faculty of Medicine, Khon Kaen University, Khon Kaen, and " Rajavithi Hospital, Bangkok, Thailand

Purpose: The coronavirus disease 2019 (COVID-19) pandemic is a global health crisis that has impacted daily life due to the policies created to contain the outbreak. Recent studies showed that medical students, a high-stress population, experienced deteriorated mental well-being during the pandemic. The aim of the present study was to assess stress and the need for support among Thai medical students during the COVID-19 pandemic, as a multicenter study.

Methods: The present study was a cross-sectional questionnaire-based study which collected data from second through sixth year medical students. Data was collected during the pandemic from multiple medical schools spanning all six regions of Thailand. Questionnaires included: demographic data; the Thai version of the Perceived Stress Scale-10 (T-PSS-10) assessing stress level and the sources of stress; and the received supports from medical schools, the satisfaction with the supports, and the further necessary needs.

Results: There were 1,395 medical students who responded to the questionnaires. Mean T-PSS-10 score was 17.8. Most of the sources of stress were related to the changing of teaching and evaluation system. Students residing in larger medical schools were significantly more satisfied with received support and tended to gain greater support than those in medium and small sized schools. Stress-relieving activities arrangement was considered the most sought after additional support by students.

Conclusion: Medical student stress levels were higher during the pandemic compared to pre-pandemic levels. Stress relieving activities, availability and access to mental health resources, and other strategies to reduce stress among medical students are urgently needed.

Key Words: Psychological stress, Medical students, COVID-19, Psychosocial support systems

\section{Introduction}

The novel coronavirus disease 2019 (COVID-19), first

found in Wuhan, China in December 2019, has spread rapidly, leading to a serious outbreak around the world. The COVID-19 outbreak has since become a global health crisis and was declared a pandemic by the World

Received: May 30, 2021 • Revised: July 18, 2021 • Accepted: July 19, 2021 Corresponding Author: Somboon Hataiyusuk (https://orcid.org/0000-0002-8775-116X) Department of Psychiatry, Siriraj Hospital, 2 Prannok Road, Bangkok, 10700, Thailand Tel: +66.2.4197373 Fax: +66.2.4197373 email: Somboon.hat@mahidol.edu
Korean J Med Educ 2021 Sep; 33(3): 203-213

https://doi.org/10.3946/kjme.2021.200

eISSN: 2005-7288

(C) The Korean Society of Medical Education. All rights reserved. This is an open-access article distributed under the terms of the Creative Commons Attribution Non-Commercial License (http:// creativecommons.org/licenses/by-nc/3.0/), which permits unrestricted non-commercial use, distribution, and reproduction in any medium, provided the original work is properly cited. 
Health Organization on March 11th 2020 [1]. Due to the high mortality rate and the rapid increase in cases worldwide, many countries have launched various policies in an effort to contain the virus. The closure of schools, shopping malls, dine-in restaurants, and other various entertainment venues, the implementation of travel restrictions, city/country lockdowns, state quarantines, and active prohibition of large gatherings have all been adopted. These measures not only impact everyday life but also require significant adjustments to this new way of living, which could cause considerable psychological stress.

Medical students are a high-stress population due to the academic competitiveness and learning outcomes required by their institutions [2-4]. During the outbreak, teaching and learning modifications put in place to maintain social distancing have vastly impacted students, resulting in increased incidences of depression and anxiety [5]. The adjustment to online learning, postponement of clinical examinations, and cancellation of clinical practice may lead to an increase in stress. A meta-analysis showed higher prevalence of insomnia, anxiety, depression, somatization, and obsessivecompulsive symptoms in health professionals during COVID-19 [6,7]. Medical students working as health care providers are at greater risk of contracting COVID-19 due to increased exposure. Worries of being infected during clinical rotation, could be another source of stress for medical students.

According to recent studies, medical students experienced clinically significant psychological stress accompanied by a deterioration in mental well-being $[8,9]$. They also reported poorer sleep and appetite than before the pandemic [10]. In a recent meta-analysis, Lasheras et al. [11] estimated the prevalence of anxiety among medical students during the pandemic to be $28 \%$ (range, 17\%-46\%). The most common stressors reported included financial concerns, impacts on daily life, effects on academic activities, and concerns with family members becoming infected [9,12]. Recent articles have discussed the psychological impact of the COVID-19 pandemic on medical students, however there is still a lack of multicenter studies in Thailand. Moreover, in order to provide the practical and direct support medical students need, assessment of the current supports provided and those that are unavailable but desperately needed, must be addressed. The aim of this multicenter study was to assess the stressors, the received support and further support needed among Thai medical students during the COVID-19 pandemic. Our research questions included "What are the level of stress and the stressors that medical students are facing during the COVID-19 pandemic?" and "What are the supports that medical students receive and need?"

\section{Methods}

The present research was a cross-sectional questionnairebased study. Fourteen medical schools were invited to take part in this study and 11 agreed to participate. Data was collected from second to sixth year medical students during the pandemic. Data spanned all six regions of Thailand, and included small, medium, and large sized medical schools. Medical school size was determined based on the total number of medical students in the academic year and was categorized accordingly. Small medical schools included those who fell below the 25th percentile, while large medical schools were those above the 75 th percentile. Those who fell between the 25th and 75th percentiles were categorized as medium. The medical education in Thailand consists of a 6-year curriculum involving preclinical years, which include the first to third year, and clinical years, which include the fourth to sixth year. First-year students were 
not included in the study since they had not yet started the academic year at the time of data collection. The online questionnaires were distributed through medical student's social media groups, student affairs offices at each medical school, and by passing the link from student to student during the pandemic from June 11th to August 27th 2020. Participants voluntarily consented to participate in the study. The questionnaires comprised three main parts. The first part obtained demographic data: sex, academic year, grade point average (GPA), religion, family income, family with stable income, social support, types of accommodation and medical school attended. The second part assessed students' stress level on the Thai version of the Perceived Stress Scale-10 (T-PSS-10) and the sources of stress using a questionnaire developed and validated by psychiatrists. The third part evaluated the support provided by medical schools, students' satisfaction with the supports, and any further support or care needed. The study was approved by the Institutional Review Board of all medical schools participating in the study (approval no., Si 487/2020, REC.63-275-3-1, PSY-2563-07395, SWUEC/E-205/2563, 157/2020, 072/2563, 25/2563, HU052/2563, RBHE C01/ 2020, 146/2563, and HE631329).

\section{Measurement tools}

\section{1) Thai version of the Perceived Stress Scale-10}

The Perceived Stress Scale (PSS) is widely used to measure the degree to which life events are perceived as stressful by asking how one perceives different aspects of one's life as uncontrollable, unpredictable, and overloading. There are 10 questions in which respondents indicate how often they have felt or thought a certain way within the past month on a 5-point Likert scale ranging from 0 (never) to 4 (very often). The total ranges from 0 to 40 with higher composite scores indicating more perceived stress. The T-PSS-10 has demonstrated good reliability and validity for estimating the level of stress perception in a Thai population with a Cronbach's $\alpha$ of 0.84 and the interclass correlation coefficient of 0.83 (95\% confidence interval, 0.722-0.881) [13]. T-PSS-10 scores were also found to positively correlate with the State Trait Anxiety Inventory and the Thai Depression Inventory and negatively correlate with the Rosenberg Self-Esteem Scale [13].

\section{2) Sources of stress}

Because the pandemic was an unprecedented event, in order to understand the issue of students' stress, the authors carried out a short interview with a focus group of 15 medical students during the pandemic, which included both clinical and preclinical year students. Based on information obtained from the interviews, researchers developed questions focusing on sources of stress most relevant to medical students. The questionnaire received content validation from two psychiatrists and encompassed 17 sources of stress, such as stress from online teaching and learning, stress from online examination and evaluation system, feeling lonely, not being able to meet friends or attend recreational activities, and fear of infection. The authors also provided an "other" option in case participants had a source of stress that was not already indicated. The participants rated each source of stress on a 4-point Likert scale ( $0=$ not stressed, $1=$ slightly stressed, 2=quite stressed, 3=very stressed). "Quite stressed" and "very stressed" were considered as stress being present and the percentage of students who were stressed in each source were reported.

\section{3) Medical schools support}

The questionnaire consisted of three questions: (1) "What kind of support does your medical school provide to students?" Answer options included "changing teaching and learning system", "changing assessment method", "financial support", "infection prevention policy and preventive equipment", "mental health care system", "stress-relieving activities arrangement", "activities to 
enhance clinical experience", "equipment support for online learning", "don't know", and "other". (2) "How satisfied you are with the support of your institution?" Responses were obtained using a 4-point Likert scale ( $3=$ very satisfied, $2=$ quite satisfied, 1=less satisfied, $0=$ not satisfied). The answers "very satisfied" and "quite satisfied" were considered as being satisfied with the support, while the answers "less satisfied" and "not satisfied" were considered as being unsatisfied. (3) "What additional support do you need?" Responses were captured using a multiple choice format and included one free response option, "stress-relieving activities arrangement", "activities to enhance clinical experience", "mental health care system", "financial support", "equipment support for online learning", "no need for additional support", and "other(s)".

\section{Statistical analysis}

Statistical analysis was conducted using PASW SPSS statistical software ver. 18.0 (SPSS Inc., Chicago, USA). Basic descriptive statistics were computed for all variables and reported as frequency and percentages for nominal/ordinal data; and means and standard deviations for continuous data. A T-test and one-way analysis of variance were performed to evaluate the associations between sociodemographic characteristics and stress level during the pandemic. All statistically significant variables $(p<0.05)$ were included in the multivariable linear regression analysis. Chi-square was used to determine the significant difference between received/ needed support and student satisfaction between various sizes of medical schools. A two-tailed $\mathrm{p}<0.05$ was accepted for statistical significance.

\section{Results}

\section{Sociodemographic data}

There were 1,395 participants (mean age \pm standard deviation $[S D]=21.03 \pm 1.70$ years) from 11 different medical schools (small 7.3\%, medium 65.5\%, and large 27.2\%). The questionnaires response rate was $16.4 \%$. Majority of the sample was female (59.4\%). Fifty-five percent of participants were in a preclinical year, while $45.2 \%$ of participants were in a clinical year. Half of the participants were living at home at the time of data collection with a quarter of them living in their own apartment and a quarter in on-campus residences. Table 1 provides the demographic data for all participants.

\begin{tabular}{|c|c|c|}
\hline & Characteristic & No. $(\%)$ \\
\hline \multicolumn{3}{|l|}{ Sex } \\
\hline Female & & $829(59.4)$ \\
\hline Male & & 552 (39.6) \\
\hline Others & & $14(1.0)$ \\
\hline \multicolumn{3}{|l|}{ Years } \\
\hline Preclinic & & 765 (54.8) \\
\hline Clinic & & 630 (45.2) \\
\hline \multicolumn{3}{|l|}{ GPA } \\
\hline$<3.00$ & & 170 (12.4) \\
\hline $3.00-3.50$ & & 535 (38.4) \\
\hline $3.51-4.00$ & & 690 (49.5) \\
\hline
\end{tabular}




\begin{tabular}{|c|c|c|c|c|c|}
\hline \multicolumn{6}{|l|}{ Table 1. (Continued) } \\
\hline & \multicolumn{2}{|c|}{ Characteristic } & & \multicolumn{2}{|r|}{ №. $|\%|$} \\
\hline \multicolumn{6}{|l|}{ Religious } \\
\hline \multicolumn{4}{|l|}{ Buddhist } & \multicolumn{2}{|c|}{$1,250(89.6)$} \\
\hline \multicolumn{4}{|l|}{ Christian } & \multicolumn{2}{|c|}{$46(3.3)$} \\
\hline \multicolumn{4}{|l|}{ Muslim } & \multicolumn{2}{|r|}{$21(1.5)$} \\
\hline \multicolumn{4}{|l|}{ Others } & \multicolumn{2}{|c|}{$78(5.6)$} \\
\hline \multicolumn{6}{|c|}{ Family income per month (US dollar) } \\
\hline \multicolumn{4}{|l|}{$<\$ 760$} & \multicolumn{2}{|c|}{$261(18.7)$} \\
\hline \multicolumn{4}{|l|}{$\$ 760-1,360$} & \multicolumn{2}{|c|}{$329(23.6)$} \\
\hline \multicolumn{4}{|l|}{$\$ 1,360-3,030$} & \multicolumn{2}{|c|}{$527(37.8)$} \\
\hline \multicolumn{4}{|l|}{$>\$ 3,030$} & \multicolumn{2}{|c|}{278 (19.9) } \\
\hline \multicolumn{4}{|l|}{ Have psychiatric disorder } & \multicolumn{2}{|c|}{$68(4.9)$} \\
\hline \multicolumn{4}{|l|}{ Have stable family income } & \multicolumn{2}{|c|}{$1,008(72.3)$} \\
\hline \multicolumn{4}{|l|}{ Have social support } & & 36 (92.8) \\
\hline Accommodation & & & & & \\
\hline Home & & & & & $17(51.4)$ \\
\hline Off-campus housing & & & & & $47(24.9)$ \\
\hline On-campus housing & & & & & $31(23.7)$ \\
\hline Sizes of medical school lc & according to $\mathrm{t}$ & number of student & schooll & & \\
\hline Small $(<250)$ & & & & & 02 (7.3) \\
\hline Medium $(250-1,000)$ & & & & & $14(65.5)$ \\
\hline Large $(>1,000)$ & & & & & $79(27.2)$ \\
\hline Table 2. Factors Related to & version of th & erceived Stress So & 10) $(N=1,3$ & & \\
\hline Variable & Mean \pm SD & Mean difference & $p$-value & $\begin{array}{l}\text { Adjusted mean } \\
\text { difference }\end{array}$ & Adjusted $p$-value \\
\hline Sex & & & & & \\
\hline Male & $17.1 \pm 5.89$ & -1.04 & 0.001 & -1.32 & $<0.001$ \\
\hline Female & $18.2 \pm 5.49$ & & & & \\
\hline With psychiatric disorder & & & & & \\
\hline Yes & $21.0 \pm 5.40$ & 3.44 & $<0.001$ & 2.59 & 0.009 \\
\hline No & $17.6 \pm 5.63$ & & & & \\
\hline Having social support & & & & & \\
\hline Yes & $17.4 \pm 5.53$ & -5.03 & $<0.001$ & -4.99 & $<0.001$ \\
\hline No & $22.4 \pm 5.42$ & & & & \\
\hline Family income per month IU & & & & & \\
\hline Less than $\$ 1,360$ & $18.5 \pm 5.80$ & 1.26 & $<0.001$ & 0.99 & 0.002 \\
\hline More than $\$ 1,360$ & $17.2 \pm 5.50$ & & & & \\
\hline Sizes of medical school & & & & & \\
\hline Small $|<250|$ & $19.2 \pm 5.95$ & 2.10 & 0.002 & 1.83 & 0.003 \\
\hline Medium $(250-1,000)$ & $17.8 \pm 5.40$ & 0.72 & 0.036 & 0.73 & 0.029 \\
\hline Large $\mid>1,000)$ & $17.1 \pm 6.11$ & & & & \\
\hline
\end{tabular}

SD: Standard deviation.

\section{Level of stress}

The mean \pm SD T-PSS-10 score was $17.8 \pm 5$.66. In order to evaluate the factors related to stress, an independent T-test was employed to compare sociodemographic data between groups. Researchers performed multivariable linear regression for all significant variables. Results showed that being female, having a 
psychiatric disorder(s), having no social support, and having low family income (less than US \$1,360/mo) were all significantly correlated with higher stress levels (Table 2). In terms of medical school size, students who attended small and medium sized medical schools had greater T-PSS-10 scores $(p=0.003)$ than those in large sized schools $(\mathrm{p}=0.029)$. There was no significant association between level of stress and religious beliefs, academic year, having a stable income, types of accommodation, or GPA. Moreover, in comparing medical schools from various regions across Thailand, whether they were in capital or non-capital cities, no significant difference in stress levels were found.

\section{Stressors}

The authors ranked the top ten stressors in medical students during the pandemic as shown in Table 3. All 10 stressors positively correlated with the T-PSS-10 scores. The two greatest concerns were "fear of system error during the exam" (54.6\%) and "uncertainty of teaching and learning arrangement" (54.6\%) followed by "lack of clinical experience" (49.7\%), "unable to learn or study effectively" (47.6\%), and "lack of practical experience (e.g., dissection in gross anatomy laboratory)" (47.3\%).

\begin{tabular}{clc}
\hline \begin{tabular}{c} 
Table 3. The Top Ten Stressors for Medical Students during the Pandemic $(N=1,395)$ \\
\hline Order
\end{tabular} & \multicolumn{1}{c}{ Issues of stress } & No. (\%) \\
\hline 1 & Fear of system error during the exam & $728(54.6)$ \\
2 & Uncertainty of teaching and learning arrangement & $728(54.6)$ \\
3 & Lack of clinical experience & $665(49.7)$ \\
4 & Unable to learn or study effectively & $644(47.6)$ \\
5 & Lack of practical experience li.e., dissection in gross anatomy) & $637(47.3)$ \\
6 & Unable to keep up with the lesson & $628(47.1)$ \\
7 & Cancellation of professional licensed exam without knowing the definite timeline & $627(47.1)$ \\
8 & Grading method adjustment & $467(35.3)$ \\
9 & Unfair online examination allowing students to cheat on a test & $414(30.8)$ \\
10 & Feeling lonely, not able to meet any friends or attend any recreational activities & $398(29.4)$ \\
\hline
\end{tabular}

Fig. 1. Types of Support Provided by Medical Schools

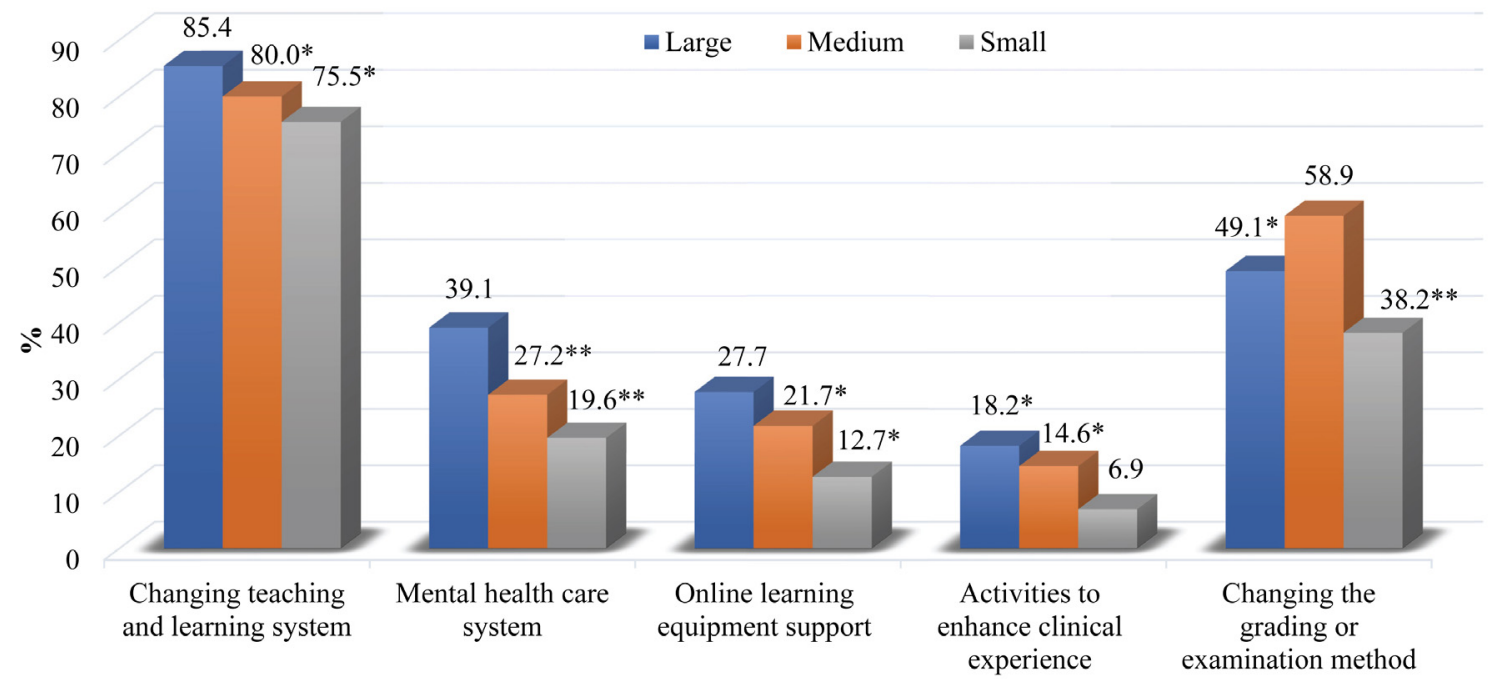

Small vs. medium vs. large medical schools.

Types of provided support

${ }^{*} p<0.05 .{ }^{* *} p<0.001$. 
Other concerns endorsed by students included the inability to keep up with the lesson, the cancellation of a professional licensed exam without knowing the definite timeline, and loneliness.

\section{Supports from medical school}

The supports provided by medical schools from most to least supportive were: "changing teaching and learning system" (81.1\%), "changing the grading or examination method" (54.7\%), "financial support" (48.8\%), "infection prevention policy and preventive equipment" (47.9\%), and "mental health care system" (29.9\%), respectively. Students from the large medical schools gained significantly greater support than those in medium and small sized medical schools in regards to "changing teaching and learning system", "mental health care system", and "online learning equipment support" ( $p<$ 0.05) (Fig. 1). Similarly, small sized medical schools offered fewer activities to enhance students' clinical experience compared to medium and large sized medical schools ( $\mathrm{p}=0.033$ and $\mathrm{p}=0.005$, respectively). However, medium sized medical schools provided significantly better support for "changing the grading or examination method" than large $(\mathrm{p}=0.001)$ and small sized medical schools $(p<0.001)$.

\section{Satisfaction with the received support}

Results found that $68.9 \%$ of students were satisfied with the support received from their medical schools. The preclinical medical students tended to have greater satisfaction than the clinical medical students $(p=0.003)$ (Fig. 2A). When comparing medical school size, medical students in large sized medical schools were more satisfied with the received supports than those in the small and medium sized medical schools $(\mathrm{p}=0.010$ and $\mathrm{p}=0.002$, respectively), as shown in Fig. $2 \mathrm{~B}$.

\section{Need for additional support}

Additional supports most needed were, "stressrelieving activities arrangement" (37.9\%), "activities to enhance clinical experience" (31.8\%), "mental health care system" (30.2\%), "financial support" (28.3\%), and "online learning equipment support" (23.3\%). Eighteen point one percent of the students reported no need for further help. In a comparison between the various sizes of medical schools, students in large sized medical schools needed less financial assistance and less online learning equipment support than those in small and medium sized
Fig. 2. Student Satisfaction with School Supports

(A)

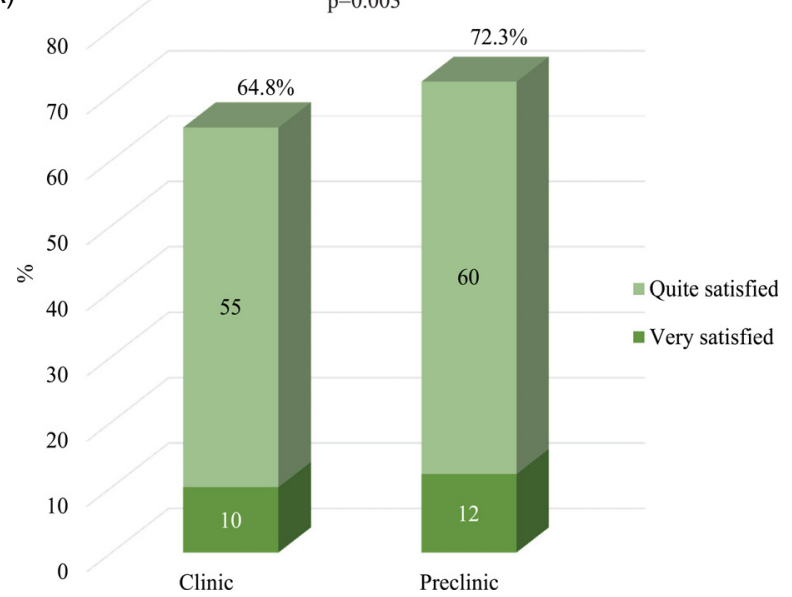

(B)

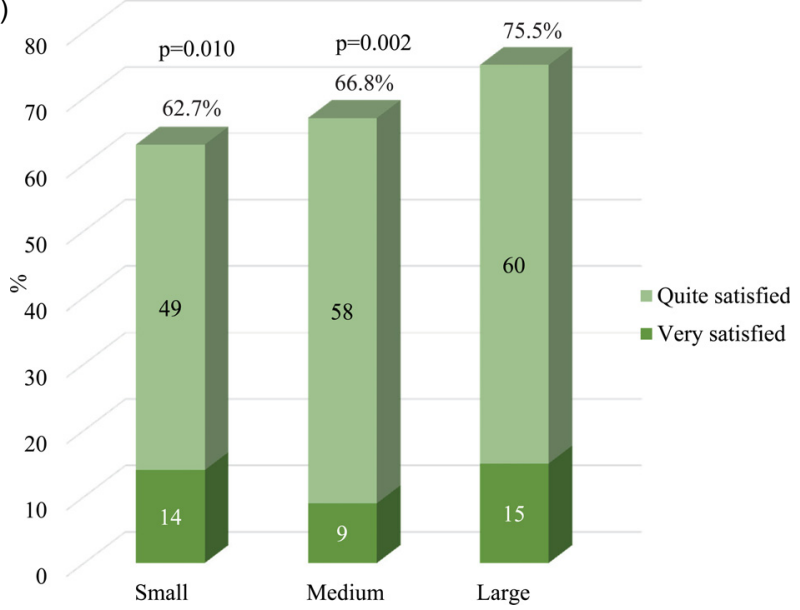

(A) Preclinical vs. clinical years. (B) Small vs. medium vs. large medical schools. 
Fig. 3. Support Needed

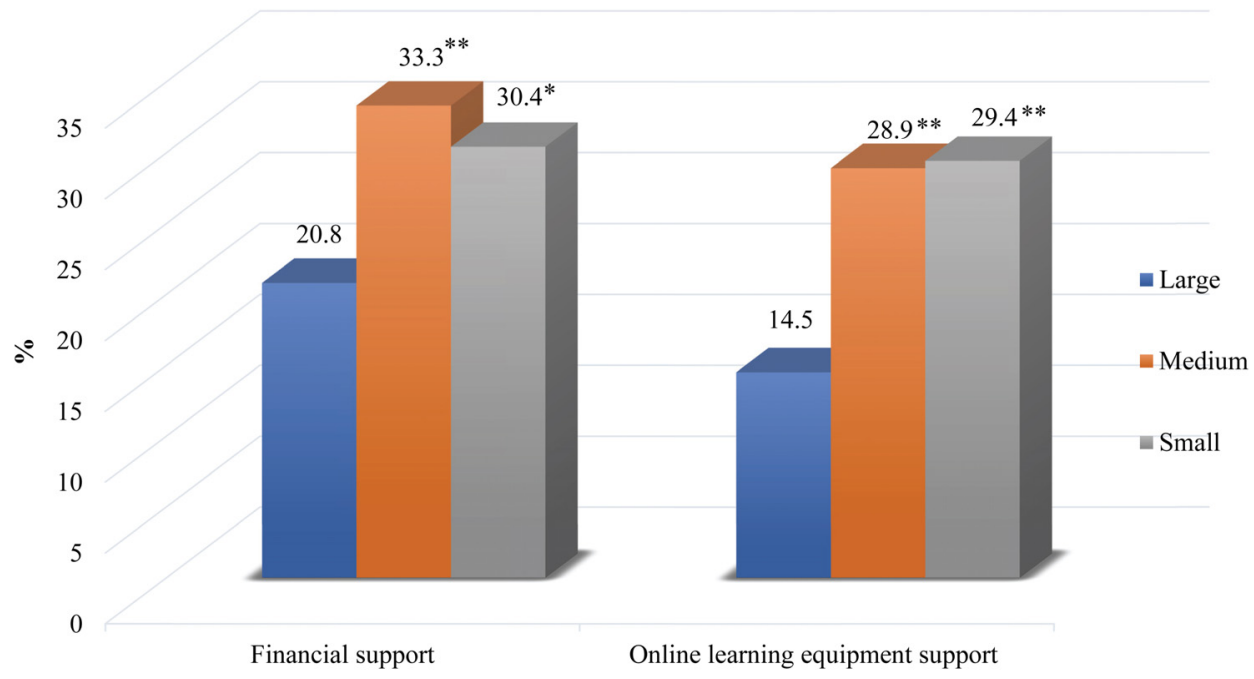

Types of further needed support

Small vs. medium vs. large medical schools.

${ }^{*} p<0.05 .{ }^{* *} p<0.001$.

medical schools $(\mathrm{p}<0.05)$ (Fig. 3). However, there was no significant difference found between medical school size and students needs for stress-relieving activities arrangement, activities to enhance clinical experience, and mental health care system.

In the narrative responses, students needed additional support with optimizing online teaching and learning methods. This might include better internet quality for both teachers and students, announcing clear teaching and learning guidelines in advance, asynchronous online lecture allowing students to review lessons after class, addressing crowded class schedules, lessening assignments, and handing out teaching materials before class. Some students also reported a need for on-campus accommodations due to a home environment unsuitable for online learning.

\section{Discussion}

The present research is the first multicenter study examining stress among medical students and the support provided by medical schools during the COVID-19 pandemic. We found that medical students are experiencing a higher level of stress compared to data collected during a usual situation in which T-PSS-10 mean \pm SD score was $13.5 \pm 4.6$ [13]. The factors most strongly correlated with higher levels of stress included being female, having a psychiatric disorder(s), absence of social support, and low family income (less than US $\$ 1,360 / \mathrm{mo}$ ), all of which were consistent with previous findings $[9,10,14]$. There was no significant difference in stress levels between medical schools located in various regions or between capital and non-capital cities. Unlike the results from a previous study, students in Wuhan, the epicenter of the pandemic, had higher anxiety levels than those in Beijing [14]. The main sources of stress identified by students, "change in teaching and learning" and "change in assessment system", were implemented in response to the country-wide lockdown and social distancing policies. Thus, the change in educational policy as a result of social distancing policies, occurred similarly in medical schools across all regions of Thailand. 
Our study found that most medical students' sources of stress were related to the changes in the teaching, learning, and evaluation system. The transition to online learning requires a substantial adjustment from students in all educational systems. Issues with online education include concerns related to in-person interaction, communication, use of technological tools, time management, experience in online education, and student assessment $[15,16]$. Moreover, in clinical year medical students, the cancellation of clinical rotation caused additional concerns regarding the acquisition of adequate clinical experience, which could significantly impact students' competencies in preparation for graduation [17]. A serious issue which would undeniably result in greater psychological stress for students.

Our results also revealed that "stress-relieving activities arrangement", "activities to enhance clinical experience", and "mental health care system" were ranked the highest needed supports by medical students. Improving student mental health and well-being was an extremely urgent priority for medical schools. Programs focused on preventing and alleviating significant stress experienced during the pandemic are absolutely essential. It should also be recognized that social distancing policies may further limit accessibility to counselling or other mental health services both for students who already have a known underlying psychiatric disease and those who do not. The modification of existing mental health services to provide telepsychiatry, virtual counselling, and online mindfulness meditation are becoming increasingly necessary. Lyons et al. [9] reported that the common activities and strategies that medical students used to successfully relieve stress during the pandemic were video chat, social media application, and mindfulness/meditation. Various stress relieving exercises and self-care activities like these need to be promoted.

Another distinctive aspect of this study was the comparison between different sizes of medical schools. We found that students in large sized medical schools had lower stress levels, derived greater satisfaction from provided support, and needed less assistance with finances and learning equipment than small and medium sized medical schools. Moreover, large sized medical schools tended to provide better support to students when it came to "changing teaching and learning system" and "mental health care system" when compared with small and medium sized schools. This might suggest that large sized medical schools, usually fortified by longestablished universities, likely have the facilities and resources to better provide students with learning materials, e-learning system support, financial aid, and access to mental health care. It is also possible that due to the infrastructure of large schools, resources are applied more promptly and effectively in the event of an emergency. Ultimately, the pandemic highlights the need for all medical schools to build up varied crucial support systems for students.

Nevertheless, our study has some limitations. First, due to the cross-sectional design, no data was collected in a usual situation pre-pandemic in order to provide a comparison. Still, the stress level from the present study was higher than the usual situation as compared with data from the previous study [13]. Second, the COVID19 pandemic is a novel situation. The situation not only increased the concern of being infected but the policies put in place to contain outbreaks also impacted daily life. The questionnaires inquiring about sources of stress and support in this study may not cover all of the possible issues or answers. However, all of the sources of stress that were gained from the focus group interviews were included and there was an "others" response option which allowed participants to provide alternate answers. Many students responded in text that they needed additional support on improving the online teaching and 
learning system, but the significance of this issue is still unknown. Even though this study was constructed only in Thailand, the COVID-19 pandemic impacted medical students worldwide, facing challenges due to the change in teaching and learning system. Our findings affirmed the high level of stress in medical students and related factors (having psychiatric disorder, no social support, and low family income) found in previous studies, which could be generalized to other countries. An interviewbased qualitative study is crucial for a deeper understanding of the issues surrounding stress, coping strategies, and additional support needed by medical students. Additionally, identifying the long-term consequences and impact of stress on medical students related to the pandemic is needed in order to prevent unfavorable effects and provide better help.

In conclusion, the stress level in medical students during the pandemic was higher than the usual situation. Most stressors were related to the changes in teaching and learning system such as online learning. Stress relieving activities, access to mental health care, and other strategies to reduce stress among medical students are urgently needed. The pandemic is a great opportunity for medical schools to strengthen their supports and allocate resources for students. Future research $\mathrm{ex}^{-}$ amining the long-term effects of stress related to the pandemic is needed.

\section{ORCID:}

Tidarat Puranachaikere: https://orcid.org/0000-0001-6768-2200;

Somboon Hataiyusuk: https://orcid.org/0000-0002-8775-116X;

Rungarun Anupansupsai: https://orcid.org/0000-0003-3652-6162;

Supinya In-iw: https://orcid.org/0000-0002-3523-4400;

Nattha Saisavoey: https://orcid.org/0000-0001-6278-3440;

Tanawat Techapanuwat: https://orcid.org/0000-0003-1779-6095;

Fasinee Arunrodpanya: https://orcid.org/0000-0003-2713-1310;
Chanchai Charonpongsuntorn: https://orcid.org/0000-0001-8710-1020;

Pakawat Wiwattanaworaset: https://orcid.org/0000-0002-3560-0415;

Areerat Siripongpan: https://orcid.org/0000-0003-0976-4026;

Wisarat Pruttithavorn: https://orcid.org/0000-0002-4504-7131;

Chayut Wonglertwisawakorn: https://orcid.org/0000-0003-1045-1887;

Pichaya Pojanapotha: https://orcid.org/0000-0001-9293-5222;

Butsayanart Rueangrong: https://orcid.org/0000-0003-2295-7236;

Nalinee Pattrakornkul: https://orcid.org/0000-0001-5665-2161;

Nontaphon Piyawattanametha: https://orcid.org/0000-0002-1372-3502;

Siriwan Piyawattanametha: https://orcid.org/0000-0002-7365-5548;

Dheeravut Ratanapichayachai: https://orcid.org/0000-0002-6222-9260

Acknowledgements: We thank Mr. Suthipol Udompunthurak, Department of Health Research and Development, Faculty of Medicine, Siriraj Hospital, Mahidol University for assistance with research analysis.

Funding: This study is supported by Siriraj Research Development Fund (managed by Routine to Research: R2R; grant no., RO16335021).

Conflicts of interest: No potential conflict of interest relevant to this article was reported.

Author contributions: TP, SH, RA, SI, NS, and TT developed the main conceptual ideas and methodological design for the study. SH, FA, CC, PW, AS, WP, CW, PP, BR, NP, NP, SP, and DR contributed to data collection and interpretation. TP and SI performed the analysis. TP took the lead in drafting the manuscript with discussion the results with all authors. All authors provided critical feedback and contributed to the final version of the manuscript. SI and NS supervised the project. All authors have approved of the final version to be published and agreed to be accountable for all aspects of the work in ensuring that questions related to the accuracy or integrity of any part of the work are appropriately resolved. 
outbreak. Psychol Med. 2020 May 11 [Epub]. https:// doi.org/10.1017/S0033291720001555.

9. Lyons Z, Wilcox H, Leung L, Dearsley O. COVID-19 and the mental well-being of Australian medical students: impact, concerns and coping strategies used. Australas Psychiatry. 2020;28(6):649-652.

10. Torun F, Torun SD. The psychological impact of the COVID-19 pandemic on medical students in Turkey. Pak J Med Sci. 2020;36(6):1355-1359.

11. Lasheras I, Gracia-García P, Lipnicki DM, et al. Prevalence of anxiety in medical students during the COVID-19 pandemic: a rapid systematic review with meta-analysis. Int J Environ Res Public Health. 2020; 17(18):6603.

12. Cao W, Fang Z, Hou G, et al. The psychological impact of the COVID-19 epidemic on college students in China. Psychiatry Res. 2020;287:112934.

13. Wongpakaran N, Wongpakaran T. The Thai version of the PSS-10: an investigation of its psychometric properties. Biopsychosoc Med. 2010;4:6.

14. Xiao H, Shu W, Li M, et al. Social distancing among medical students during the 2019 coronavirus disease pandemic in China: disease awareness, anxiety disorder, depression, and behavioral activities. Int J Environ Res Public Health. 2020;17(14):5047.

15. Esani M. Moving from face-to-face to online teaching. Clin Lab Sci. 2010;23(3):187-190.

16. Rajab MH, Gazal AM, Alkattan K. Challenges to online medical education during the COVID-19 pandemic. Cureus. 2020;12(7):e8966.

17. Choi B, Jegatheeswaran L, Minocha A, Alhilani M, Nakhoul M, Mutengesa E. The impact of the COVID-19 pandemic on final year medical students in the United Kingdom: a national survey. BMC Med Educ. 2020; 20(1):206.

8. Li Y, Wang Y, Jiang J, et al. Psychological distress among health professional students during the COVID-19 